\title{
Vaccinations, infections and antibacterials in the first grass pollen season of life and risk of later hayfever
}

\author{
S. A. Bremner*, I. M. Carey*, S. DeWilde* , N. Richards ${ }^{\dagger}$, W. C. Maier ${ }^{\ddagger}$, S. R. Hilton* , D. P. Strachan* and D. G. Cook ${ }^{*}$ \\ *Division of Community Health Sciences, St George's, University of London, London, UK, ${ }^{\dagger}$ Cegedim Strategic Data, Send, Surrey, UK and ${ }^{\ddagger}$ Elan Pharma Ltd, Stevenage, \\ Herts, UK
}

\section{Clinical and Experimental Allergy}

Correspondence:

Derek G. Cook, Division of Community Health Sciences, St George's, University of London, Cranmer Terrace, London SW17 ORE, UK.

E-mail:d.cook@sgul.ac.uk

\begin{abstract}
Summary
Background It has been hypothesized that early-life exposure to vaccinations, infections or antibacterials influence allergic disease development. Concurrent exposure to grass pollens may alter any effect.

Objective To test the hypothesis that exposure to antibacterials, vaccinations (DTP or MMR) or specific infections during the first grass pollen seasons of life influences the risk of hayfever more than at any other time of the year.

Methods Nested case-control studies were based on birth cohorts within two large databases of computerized patient records from UK general practices: the General Practice Research Database (GPRD) and Doctors' Independent Network (DIN). Seven thousand ninety-eight hayfever cases, diagnosed after age 2, were matched to controls for practice, age, sex and follow-up of control to case ascertainment date. Conditional logistic regression was used to compare exposure by age 1 (age 2 for MMR) inside vs. outside the grass pollen season (May, June, July). Odds ratios (ORs) were pooled across databases.

Results There were no associations in either database between MMR during vs. outside the grass pollen season and later hayfever. Of 23 infections studied, none were statistically significant; although analyses for the less common conditions were limited by low statistical power. The pooled OR for hayfever comparing exposure to antibacterials only in the grass pollen season with only outside it was 1.20 (95\% CI 0.98-1.47) and for DTP was 0.84 (95\% CI 0.72-0.98).

Conclusion Although an interaction between early exposure to microbial agents and concurrent grass pollen exposure on hayfever risk seemed plausible, there was little evidence to support it across a range of analyses. However, the effect of DTP though weak deserves further study.
\end{abstract}

Keywords antibacterial, grass pollen allergy, infection, primary care databases, vaccination Submitted 1 November 2006; revised 15 December 2006; accepted 7 February 2007

\section{Introduction}

The earliest grass pollen seasons of a child's life are potentially critical periods for the initiation and development of pollen sensitization and hayfever. Previous epidemiological studies have explored this by investigating variations in the prevalence of pollen allergy by month of birth, but the results have been inconsistent [1-7]. In 755 Japanese schoolchildren, the prevalence of allergic rhinitis (AR) was significantly higher in those who were born between August and October [4]. In 6535 10-year- olds in Munich and Bavaria, those born in May had a 50\% increased risk of hayfever whereas those born in November were protected (40\% reduced risk) [5]. In a study of Derbyshire schoolchildren, there was no significant association between month of birth and hayfever prevalence, although prevalence was the lowest for June and July births [7]. More specifically, it has been hypothesized that concurrent challenge to the immune system by pollens and microbial agents early in life may inhibit proliferation of T-helper type 2 (Th2) cell clones and thereby prevent allergy [8]. In the only study to test this hypothesis 
epidemiologically, Strachan et al. [6] studied 11765 adolescent children in Sheffield, UK, and found no relationship between infection in month 1 and the prevalence of later hayfever in 960 June births.

Previously, we and others have exploited the potential of large primary care databases to examine the role of factors operating in the first year of life on the risk of developing hayfever and asthma. The roles of routine vaccination [9, 10], infectious illnesses [11] and antibacterials $[11,12]$ have been examined. Overall, the findings have been negative; however, the possibility exists that exposure to these agents during the grass pollen season may induce different effects from exposure outside the grass pollen season.

We therefore hypothesized that: (i) routine vaccination, (ii) infectious illnesses presenting during the grass pollen season or (iii) exposure to antibacterials during the grass pollen season, indicating infectious illness at the time of early allergen exposure, reduce the risk of subsequent hayfever. We investigate these hypotheses using two large UK-based primary care databases (PCDs): the General Practice Research Database (GPRD) and the Doctors' Independent Network (DIN) [13, 14]. The advantage of using these is that the exposures and outcome are reliably recorded, the results apply to the general population and the analyses are based on large numbers of subjects. The latter is particularly important when examining the interaction between microbial agents and the grass pollen season.

\section{Methods}

We conducted nested case-control studies within a GPRD cohort of 76310 children born between 1989 and 1993 from 464 practices and within a DIN cohort of 40183 children born between 1989 and 1997 from 141 general practices. All children had at least 5 years of follow-up from birth and had been registered with the practice within 3 months of birth. Background information on the DIN database and the birth cohorts defined in GPRD and DIN are published elsewhere [13, 14].

For each case of hayfever diagnosed, we selected one control child, matched for general practice, sex, birth month and follow-up of control to at least date of diagnosis of case. A more detailed account can be found in Bremner et al. [12]. Cases of hayfever were those who had diagnostic codes and/or treatment for hayfever, after 2 years of age. Children who were only diagnosed before age 2 and not subsequently confirmed, or who only ever had non-specific AR codes that did not exhibit a seasonal pattern were excluded from being cases or controls.

The advantages of using the two separate databases were: (i) we could assess the consistency of results across databases and (ii) pooling data from both databases increased power. Pragmatically, we defined the grass pollen season as May, June and July, although we recognize that this varies according to location and annual weather fluctuations [15]. May, June and July births are likely to be exposed to two partial grass pollen seasons in year 1 .

We carried out three different matched case-control analyses, all analysed by conditional logistic regression using proc phreg in SAS version 8.1 [16]. In the first, we considered: (i) only those pairs where both infants were fully vaccinated to third DTP by month 12 , exposure being at least one dose within the grass pollen season vs. no doses within the grass pollen season; (ii) only those case-control pairs with both members vaccinated with MMR by 24 months, with exposure being MMR within vs. outside a pollen season. We excluded pairs where the case and/or control were not exposed because such non-exposed subjects may differ from those exposed in important respects such as consultation behaviour, social circumstances or family size.

In the second analysis, we explored a wide variety of infections ranging from common, such as diarrhoea and vomiting, to rare, such as threadworms, as defined in DeWilde et al. [17]. As a control condition, we also included behavioural problems in our selection, as these are not uncommon and could not feasibly be related to the risk of developing hayfever. We were interested in comparing the effect upon hayfever of infection only in a grass pollen season vs. the same infection only outside a grass pollen season. However, for many of the casecontrol pairs where one child fell into one of these two exposure groups, the other would either not be exposed at all or be exposed in both periods. For example those case-control pairs where one was exposed in the pollen season and the other was not exposed at all provide an estimate of pollen season exposure vs. no exposure. Equally, other pairs where one was exposed outside the pollen season and the other was not exposed provide an estimate of non-pollen season exposure vs. no exposure. Putting both estimates together gives an estimate of pollen vs. non-pollen season exposure. Excluding all such pairs would exclude the majority of subjects for certain conditions, severely compromising statistical power. For infections, we therefore based the analysis on all casecontrol pairs.

In the third analysis, exposures to antibacterials in these months were flagged. We then defined variables to code exposure only in the grass pollen season, only outside it, in both periods and in neither period of the first 12 months of life. As with immunizations, the analysis was limited to those case-control pairs where both were exposed to antibacterials during the first year of life.

Odds ratios (ORs) were pooled across databases using a fixed-effect model. This was carried out using the metaanalysis command available for Stata [18, 19]. GPRD's 
Table 1. Odds ratios of hay fever given exposure to antibacterials, DTP or infection (by 12 months) or MMR (by 24 months) in a grass pollen season vs. outside one

\begin{tabular}{|c|c|c|c|c|c|c|c|c|}
\hline \multirow[b]{2}{*}{ Exposure } & \multicolumn{4}{|c|}{ Totals (cases + controls) } & \multirow[b]{2}{*}{ Exposed (\%) } & \multicolumn{3}{|c|}{ Pooled across GPRD and DIN } \\
\hline & Not $^{*}$ & out & in & both & & $\mathrm{OR}$ & $95 \% \mathrm{CI}$ & $P$-value \\
\hline $\mathrm{DTP}^{\dagger}$ by 12 months & 2018 & 6126 & 6052 & N/A & 85.8 & 0.84 & $(0.72,0.98)$ & 0.02 \\
\hline $\mathrm{MMR}^{\ddagger}$ by 24 months & 673 & 9690 & 3833 & $\mathrm{~N} / \mathrm{A}$ & 95.3 & 1.05 & $(0.94,1.18)$ & 0.38 \\
\hline \multicolumn{9}{|l|}{ Infection ${ }^{\ddagger}$ by 12 months } \\
\hline Upper respiratory tract & 7510 & 4667 & 799 & 1220 & 47.1 & 1.02 & $(0.88,1.19)$ & 0.78 \\
\hline Conjunctivitis & 9676 & 3156 & 870 & 494 & 31.8 & 1.04 & $(0.89,1.20)$ & 0.65 \\
\hline Diarrhoea and vomiting & 10034 & 3077 & 695 & 390 & 29.3 & 1.01 & $(0.86,1.20)$ & 0.86 \\
\hline Acute otitis media & 11091 & 2334 & 463 & 308 & 21.9 & 0.95 & $(0.78,1.17)$ & 0.65 \\
\hline Bronchitis and chest infection & 12018 & 1668 & 302 & 188 & 15.2 & 1.17 & $(0.91,1.49)$ & 0.22 \\
\hline Candidiasis & 11783 & 1660 & 604 & 149 & 17.0 & 1.02 & $(0.84,1.23)$ & 0.84 \\
\hline Viral illness & 13175 & 737 & 242 & 42 & 7.2 & 0.97 & $(0.72,1.30)$ & 0.83 \\
\hline Fever/pyrexia & 13233 & 630 & 269 & 64 & 6.8 & 1.05 & $(0.78,1.41)$ & 0.73 \\
\hline Sore throat & 13438 & 553 & 171 & 34 & 5.3 & 0.88 & $(0.66,1.17)$ & 0.38 \\
\hline Bronchiolitis & 13329 & 767 & 69 & 31 & 6.1 & 0.86 & $(0.52,1.42)$ & 0.56 \\
\hline Laryngitis and croup & 13688 & 403 & 93 & 12 & 3.6 & 1.10 & $(0.70,1.74)$ & 0.67 \\
\hline Chickenpox & 13757 & 314 & 123 & 2 & 3.1 & 1.14 & $(0.74,1.75)$ & 0.55 \\
\hline Other viral rash & 13938 & 171 & 82 & 5 & 1.8 & 0.91 & $(0.54,1.53)$ & 0.72 \\
\hline Impetigo & 14016 & 128 & 47 & 5 & 1.3 & 1.64 & $(0.75,3.59)$ & 0.22 \\
\hline Otitis externa & 14069 & 87 & 37 & 3 & 0.9 & 0.50 & $(0.22,1.10)$ & 0.09 \\
\hline Chronic otitis media & 14076 & 95 & 24 & 1 & 0.8 & 0.74 & $(0.30,1.84)$ & 0.52 \\
\hline Measles & 14058 & 105 & 33 & 0 & 1.0 & 1.71 & $(0.76,3.84)$ & 0.20 \\
\hline Rubella & 14047 & 103 & 45 & 1 & 1.0 & 0.67 & $(0.33,1.36)$ & 0.26 \\
\hline Urinary tract infections & 14039 & 111 & 35 & 11 & 1.1 & 0.69 & $(0.35,1.38)$ & 0.30 \\
\hline Threadworms & 14119 & 58 & 19 & 0 & 0.5 & 1.03 & $(0.36,2.96)$ & 0.96 \\
\hline Influenza & 14060 & 121 & 14 & 1 & 1.0 & 0.83 & $(0.27,2.58)$ & 0.75 \\
\hline Dermatophytosis & 14120 & 60 & 15 & 1 & 0.5 & 0.56 & $(0.16,1.98)$ & 0.37 \\
\hline Abscess/boil/nail infection & 14135 & 40 & 19 & 2 & 0.4 & 2.18 & $(0.65,7.37)$ & 0.21 \\
\hline \multicolumn{9}{|l|}{ Control condition } \\
\hline Behavioural problems & 13616 & 412 & 145 & 23 & 4.1 & 1.03 & $(0.70,1.51)$ & 0.88 \\
\hline Antibacterials ${ }^{\ddagger}$ by 12 months & 5491 & 5618 & 740 & 2347 & 61.3 & 1.20 & $(0.98,1.47)$ & 0.07 \\
\hline
\end{tabular}

For the vaccinations and antibacterials, case-control pairs were restricted to those where both had been exposed.

*“Not" exposed to antibacterials and vaccinations were not analysed but are presented for completeness.

${ }^{\dagger}$ Any DTP dose in grass pollen season vs. none in grass pollen season.

${ }^{\ddagger}$ In grass pollen season versus outside grass pollen season only.

Totals (cases+controls) = across GPRD and DIN; number in each exposure category, ignoring matching none = unexposed; out = exposed only outside grass pollen season; in = exposed only inside grass pollen season; both = exposed both out- and inside grass pollen season.

GPRD, General Practice Research Database; DIN, Doctors' Independent Network; OR, odds ratio; CI, confidence interval, N/A, not applicable.

Scientific and Ethics Advisory Group (SEAG) gave ethical approval for our study.

\section{Results}

The cumulative incidence of hayfever in GPRD was 5.6\% and 7.3\% in DIN. In GPRD, the cases were followed up for a mean of 7.4 years, and the mean age of first diagnosis of hayfever was 4.6 years. The corresponding figures in DIN were 8.2 years of follow-up and a mean age of 5.1 years at first diagnosis. Further details are available in Bremner et al. [12]. In both our GPRD and our DIN cohorts, the highest percentages of hayfever cases had been born in March, April, May and June (around 9-10\% in each of these months). It should be noted that season of birth is controlled for in our study design by matching of controls to cases for date of birth.

To illustrate the level of exposure to the different microbial agents by season, we give the total number of cases plus controls in both cohorts (ignoring matching): (i) not exposed, (ii) exposed only outside the grass pollen season, (iii) exposed only within the grass pollen season and (iv) exposed both in and outside the grass pollen season as well as the percentage of cases plus controls exposed (Table 1).

\section{Vaccinations}

In GPRD, 83.7\% of case-control pairs received their third DTP before age 1 and 94.7\% received their MMR before 
Risk of later hay fever given exposure to vaccinations, infections and antibacterials (inside vs, outside the grass pollen season): (G)=GPRD (D)=DIN

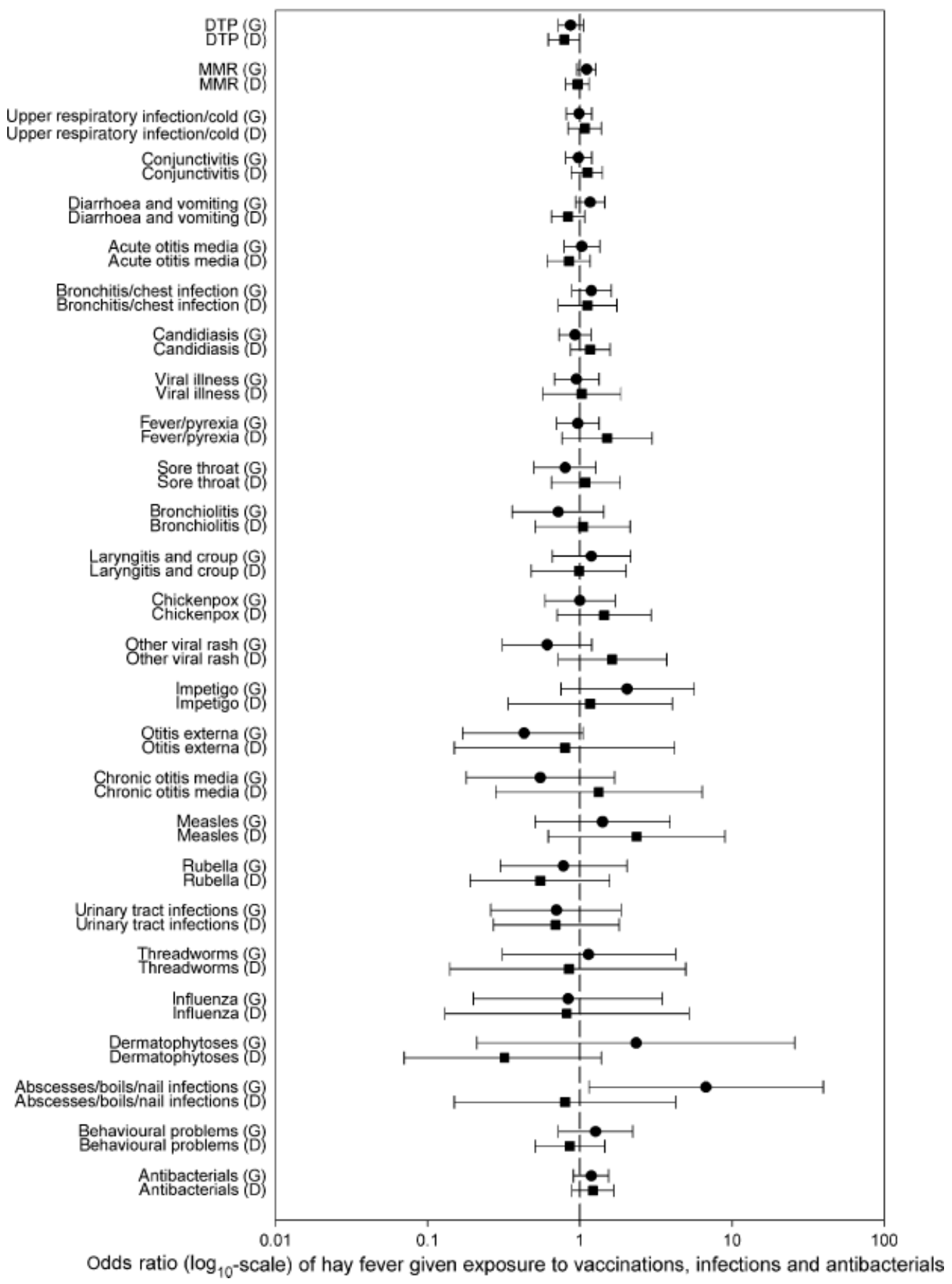

Figure 1. Risk of later hayfever, given exposure to vaccinations, infections and antibacterials (inside vs. outside the grass pollen season): $(\mathrm{G})=\mathrm{General}$ Practice Research Database (D) = Doctors' Independent Network odds ratio ( $\log _{10}$ scale) of hayfever, given exposure to vaccinations, infections and antibacterials.

age 2. In DIN, the percentages were $88.8 \%$ and $96.1 \%$. Our analyses were restricted to the case-control pairs where both received vaccination in the relevant time period.

Having any dose of DTP during the grass pollen season (compared with DTP outside the grass pollen season) was associated with a small reduction in the OR of hayfever in both GPRD [OR $=0.87,95 \%$ confidence interval (CI)
$0.72-1.06]$ and in DIN (OR $=0.79,95 \%$ CI 0.62-1.00) (Fig. 1) The fixed-effects pooled estimate was 0.84 (95\% CI 0.72-0.98) (Table 1).

Receipt of MMR in a grass pollen season by age 24 months was associated with an increased OR of hayfever in the GPRD (1.11) and a reduced OR in DIN (0.97) (Fig. 1). Both the CI estimates included 1 and the fixed-effects 
model gave a combined estimate of 1.05, which was not statistically significant (Table 1 ).

\section{Infections}

The prevalence of infections among all case-control pairs ranges from $47 \%$ for URT to $0.4 \%$ for abscesses (Table 1 ). As a result, the power for contrasting risk of exposure within to outside the groups is limited for a number of the infections. This is reflected in wider confidence limits for the estimated ORs, particularly when analysing the two databases separately (Fig. 1).

For some conditions, there was substantial variability between the GPRD and DIN results (Fig. 1). For most conditions, however, the overlap between the CIs was large and, of the 23 infections plus behavioural problems comparable in this way, in only seven instances did the estimate from one database not lie within the 95\% CI of the other database. In 14 comparisons, one database estimate was negative $(\mathrm{OR}<1)$ and the other positive $(\mathrm{OR}>1)$. There was no tendency for one database to consistently report higher ORs than the other (Fig. 1).

On pooling, most of the ORs were close to 1 (Table 1). The CIs were wide for many of the conditions, even after pooling. The only border line significant association with hayfever was for otitis externa within compared with outside the grass pollen season (pooled OR 0.50, 95\% CI $0.22-1.10, P=0.09$ ).

\section{Antibacterials}

In GPRD, for cases and controls combined, 5.1\% of children were exposed to antibacterials only in a grass pollen season, $17.9 \%$ were exposed both within and outwith a grass pollen season, 40.3\% were exposed only outside and 36.7\% were not exposed. In DIN, the percentages were 5.4\%, 14.5\%, 38.6\% and 41.5\%.

Our analyses were restricted to the case-control pairs where both members had received antibacterials in year 1 (43.6\% in GPRD and 36.6\% in DIN). The OR for exposure within compared with outside the grass pollen season was 1.19, 95\% CI 0.91-1.54) in GPRD and 1.22 (95\% CI 0.89-1.67) in DIN (Fig. 1). The pooled OR was 1.20 (95\% CI 0.98-1.47) (Table 1).

\section{Discussion}

In two population-based birth cohorts, we have not demonstrated any significant relationship between hayfever and vaccination with MMR, with infections or use of antibacterials relative to the grass pollen season. The significant effect of DTP may be a chance finding, given the large number of comparisons made, but given its policy relevance it deserves further study.
In investigating exposure to early-life infections and risk of subsequent atopic disease, some authors have considered the total burden of infection [20-23]. We decided not to define the total burden as we were investigating such a broad range of conditions and because of the difficulty of distinguishing new disease episodes. While incident infections are easy to identify in PCDs, recurrent diagnostic codes may not reflect new disease occurrences.

Despite differences in coding conventions and data structure, the two databases revealed similar results, instilling greater confidence in the generalizability of our conclusions. Compared with our earlier publications, which examined the effect of exposure to antibacterials or immunizations at any time in the first year of life vs. not, the analyses presented here are less likely to be confounded [10, 12]. This is because, unlike our earlier studies, we are not comparing subjects exposed or not exposed to a particular microbial agent, which may be confounded with individual and family characteristics, such as consultation behaviour. Rather, we are comparing those exposed in different seasons. Such differences are unlikely to be confounded by individual or family characteristics.

We considered hayfever to be a suitable outcome to investigate as it is undeniably atopic and the relevant allergen exposure is confined to the spring and summer months. Further, the crucial development period of the immune system investigated here (largely year 1) and the diagnosis of hayfever have substantial temporal separation. We carefully defined treated hayfever using specific therapies and seasonal presentation, and believe the vast majority of our cases to have hayfever. Criticism could be levelled at our definition of the pollen season; some grass pollens are still being released in August, a month that we did not include in our definition of the pollen season, but these are at much lower levels than in May, June and July [24]. The effect of this would be a reduction in power to detect an OR different to 1 for the comparison 'exposed only in the grass pollen season' vs. 'exposed only outside the grass pollen season'.

Our study posed a well-defined question, with minimal confounding, across a range of exposures that are central to the 'hygiene hypothesis'. While previous studies have investigated early exposure to the agents we describe in relation to hayfever, only one has addressed this interaction with the pollen season [6]; few others would have had sufficient statistical power to do so. Although the notion of an interaction between microbial agents and the grass pollen season on hayfever risk seemed plausible, there was little evidence to support it across a range of analyses. We conclude that previous studies that considered only allyear exposures were not misleading. However, given its policy relevance, the borderline effect of DTP deserves further study. 


\section{Acknowledgements}

This work was funded by Wellcome Trust Grant 065177/ Z/01/Z.

Conflict of interest: N. R. is director of Cegedim Strategic Data Ltd which markets DIN data to pharmaceutical companies.

\section{References}

1 Aberg N. Birth season variation in asthma and allergic rhinitis. Clin Exp Allergy 1989; 19:643-8.

2 Schafer T, Przybilla B, Ring J, Kunz B, Greif A, Uberla K. Manifestation of atopy is not related to patient's month of birth. Allergy 1993; 48:291-4.

3 Sibbald B, Rink E. Birth month variation in atopic and nonatopic rhinitis. Clin Exp Allergy 1990; 20:285-8.

4 Saitoh Y, Dake Y, Shimazu S et al. Month of birth, atopic disease, and atopic sensitization. J Invest Allergol Clin Immunol 2001; 11:183-7.

5 Wjst M, Dold S, Reitmeir P, Stiepel E, von Mutius E. Month of birth and allergic disease at the age of 10. Clin Exp Allergy 1992; 22:1026-31.

6 Strachan DP, Taylor EM, Carpenter RG. Family structure, neonatal infection, and hay fever in adolescence. Arch Dis Child 1996; 74:422-6.

7 Anderson HR, Bailey PA, Bland JM. The effect of birth month on asthma, eczema, hayfever, respiratory symptoms, lung function, and hospital admissions for asthma. Int J of Epidemiol 1981; 10:45-51.

8 Martinez FD. Role of viral infections in the inception of asthma and allergies during childhood: could they be protective? Thorax 1994; 49:1189-91.

9 McKeever TM, Lewis SA, Smith C, Hubbard R. Vaccination and allergic disease: a birth cohort study. Am J Public Health 2004; 94:985-9.

10 Bremner SA, Carey IM, DeWilde S et al. Timing of routine immunisations and subsequent hay fever risk. Arch Dis Child 2005; 90:567-73.

11 McKeever TM, Lewis SA, Smith C et al. Early exposure to infections and antibiotics and the incidence of allergic disease: a birth cohort study with the West Midlands General
Practice Research Database. J Allergy Clin Immunol 2002; 109: 43-50.

12 Bremner SA, Carey IM, DeWilde S et al. Early-life exposure to antibacterials and the subsequent development of hayfever in childhood in the UK: case-control studies using the General Practice Research Database and the Doctors' Independent Network. Clin Exp Allergy 2003; 33:1518-25.

13 Carey IM, Cook DG, DeWilde S et al. Developing a large electronic primary care database (Doctors' Independent Network) for research. Int J Med Inform 2004; 73:443-53.

14 Carey I, Cook D, DeWilde S et al. Implications of the problem orientated medical record (POMR) for research using electronic GP databases: a comparison of the Doctors Independent Network Database (DIN) and the General Practice Research Database (GPRD). BMC Family Practice 2003; 4:14.

15 Emberlin J, Mullins J, Corden J et al. Regional variations in grass pollen seasons in the UK, long-term trends and forecast models. Clin Exp Allergy 1999; 29:347-56.

16 SAS Institute Inc. SAS System for SunOS, version 8.1. Cary, NC, USA: SAS Institute Inc, 1999.

17 DeWilde S, Carey IM, Bremner SA et al. A comparison of the recording of 30 common childhood conditions in the Doctors' independent network and the general practice research databases. Health Stat Quart 2004; 22:21-30.

18 Stata Corporation. Stata for Windows, version 8.0. College Station, TX, USA: Stata Corporation, 1985-2003.

19 Sharp S, Sterne J. Sbe16: meta-analysis. Stata Techn Bull 1997; 38:9-14.

20 Bager P, Westergaard T, Rostgaard K, Hjalgrim H, Melbye M. Age at childhood infections and risk of atopy. Thorax 2002; 57:379-82.

21 Benn CS, Melbye M, Wohlfahrt J, Bjorksten B, Aaby P. Cohort study of sibling effect, infectious diseases, and risk of atopic dermatitis during first 18 months of life. BMJ 2004; 328:1223.

22 Cullinan P, Harris JM, Taylor AJN et al. Can early infection explain the sibling effect in adult atopy? Eur Respir J 2003; 22:956-61.

23 Paunio M, Peltola H, Virtanen M, Leinikki P, Makela A, Heinonen OP. Acute infections, infection pressure, and atopy. Clin Exp Allergy 2006; 36:634-9.

24 Emberlin J, Savage M, Jones S. Annual variations in grass pollen seasons in London 1961-1990: trends and forecast models. Clin Exp Allergy 1993; 23:911-8. 